\title{
AIAA 99-0309
}

\section{Impact of Thermal Gradients on Wind Tunnel Force Measurements}

\author{
James Hereford \\ Christopher Newport University, Newport News, VA \\ Peter A. Parker and Ray D. Rhew \\ NASA Langley Research Center, Hampton, VA
}

37th AIAA Aerospace Sciences

Meeting and Exhibit

January 11-14, 1999

Reno, Nevada 


\title{
Impact of Thermal Gradients on Wind Tunnel Force Measurements
}

\author{
James Hereford, Christopher Newport University, Newport News, VA \\ and \\ Peter A. Parker, Ray D. Rhew, NASA Langley Research Center, Hampton, VA
}

\begin{abstract}
In a wind tunnel facility, the direct measurement of forces and moments induced on the model are performed by a force measurement balance. The measurement balance is a precision-machined device that has strain gages at strategic locations to measure the strain (i.e., deformations) due to applied forces and moments. The strain gages convert the strain (and hence the applied force) to an electrical voltage that is measured by external meters.

Thermal gradients can complicate the process, however. Thermal gradients on the balance cause differential expansion (or contraction) of various parts of the balance that induce a strain that is detected by the strain gages and is indistinguishable from an external applied force. The thermal gradients can result when testing is done at elevated temperatures or at cryogenic temperatures such as at the National Transonic Facility (NTF) at NASA Langley Research Center (LaRC).

The thermal gradients will impact wind tunnel testing in several ways. First, they will affect the quality of certain measurements. At the NTF, the affects are most pronounced on the

Uncompensated outputs due to thermal gradients can be as much as eight times the specified accuracy of the measurement balance. Second, thermal gradients affect wind tunnel productivity. With present compensation schemes, the users must wait (at great expense) until the balance is in quasi-thermal equilibrium before taking data. Third, compensating for thermal gradients requires additional components on the balance thus increasing the on-board instrumentation.

NASA LaRC has initiated a program to find a more effective method for compensating for thermal gradients. The program (ThermallyInduced Gradient Effects Research, TIGER) places temperature sensors on the measurement balance to determine the appropriate thermal gradients and then factor out their effects. Part of the research, however, is to determine how many temperature sensors are necessary and in which locations. To help answer these question, a Genetic Algorithm (GA) was developed to analyze data from the balance and determine the most important temperature sensors. This paper presents results from the GA as well as issues/problems that the GA output raises.
\end{abstract} axial force or drag measurement.

\section{Introduction}

In a wind tunnel facility, the direct measurement of forces and moments induced on the model are performed by a force measurement balance. (An example of a balance is shown in Figure 1.) The measurement balance is a precision-machined device that has strain gages at strategic locations to measure the strain (i.e., deformations) due to applied forces and moments. The strain gages convert the strain (and hence the applied force) to an electrical voltage that is measured by external meters. 


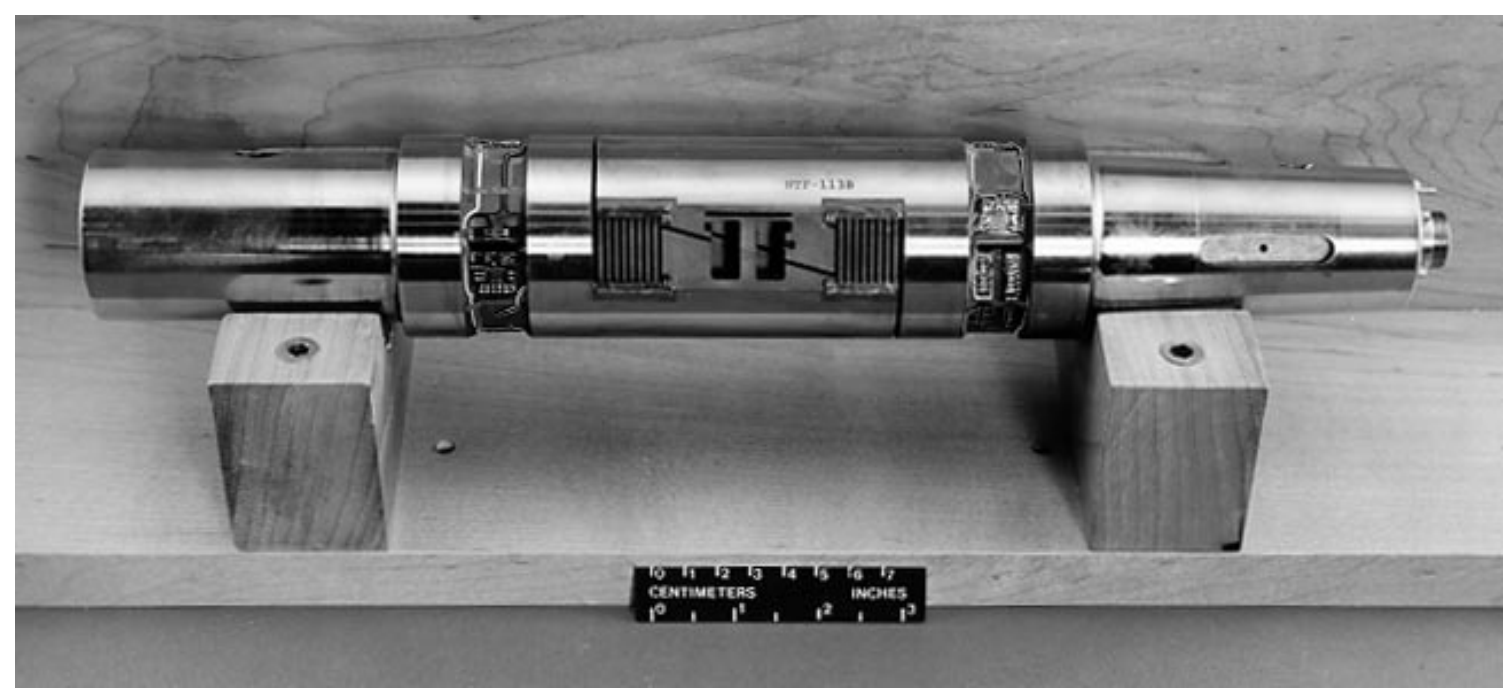

Figure 1: Picture of balance (NTF-113B) used at National Transonic Facility

Different wind tunnels have different test conditions. For example, the National Transonic Facility (NTF) located at NASA Langley Research Center is a complex wind tunnel facility in terms of capability and conditions. The NTF is a fan driven, closed circuit, continuous flow, pressurized wind tunnel. The NTF test gas may be air or nitrogen. By using liquid nitrogen as a coolant, the tunnel test temperature range is variable from $-155^{\circ} \mathrm{C}$ to $65^{\circ} \mathrm{C}\left(-250^{\circ} \mathrm{F}\right.$ to $\left.150^{\circ} \mathrm{F}\right)$. Conventional wind tunnels, such as the 16 foot tunnel at NASA-LaRC, have a test temperature range from $20^{\circ} \mathrm{C}$ to about $75^{\circ}$ $\mathrm{C}\left(70-170^{\circ} \mathrm{F}\right)$. At the other extreme, hypersonic wind tunnels have a temperature range of $315^{\circ} \mathrm{C}\left(600^{\circ} \mathrm{F}\right)$ and the balance can get as hot as $120^{\circ} \mathrm{C}\left(250^{\circ} \mathrm{F}\right)$.

The measuring of forces and moments on wind tunnel models is more challenging at non-ambient (cryogenic or elevated) temperatures due to strains caused by differential thermal expansion. Current correction techniques for thermal gradient effects will be discussed in Section 2. Section 3.1 will discuss an on-going research project at NASALaRC to develop a better compensation/correction technique for thermal gradient effects. Part of that research was the development of a Genetic Algorithm to aid in analyzing the data which will be discussed in Sections 3.2 through 3.4.

\section{Impact of Thermal Gradients}

\subsection{General Observations}

Several general comments about thermal gradients on force measurement balances:

- Thermal gradients do occur and can be quite large. In an environment like the National Transonic Facility, temperature gradients of as much as $45^{\circ} \mathrm{C}\left(80^{\circ} \mathrm{F}\right)$ can appear on the force measurement balance. In more conventional wind tunnels, the 
temperature gradients can be as much as $17^{\circ} \mathrm{C}\left(30^{\circ} \mathrm{F}\right)$. (For conventional wind tunnels, the temperature of the tunnel gas rises due to heat from the fan motors.)

- Thermal gradients on the balance cause real strain. That is, varying temperatures on the balance will cause the balance to bend and deform and thus producing strain on the balance. This strain occurs because of differential expansion (or contraction) of the balance due to the different temperatures. One corollary: because the thermal gradients produce real strain, the impact of the gradients at the strain gage sensor is indistinguishable from an external force.

- Thermal gradients primarily affect the axial force measurement. This results from a combination of (a) the design of the balance and (b) the axial (drag) measurement is usually more sensitive than the other measurements such as normal.

- Awareness of the impact of thermal gradients is not new and not confined to NASA. Technical papers from as early as 1961 discuss the "possible effects of temperature gradients on zero drift". ${ }^{1}$ The Dutch authors describe using strips of copper wire to ensure no zero drift for a uniform rise in temperature but acknowledge that the compensation can make the problem worse if there is a temperature gradient. A more recent Dutch paper has the following quotes:

"The drag element as a whole, being a redundant parallelogram construction, is susceptible to temperature gradients which cannot be compensated for with clever strain-gauge bridge configurations. Bridge compensation with temperature senosrs, positioned strategically all over the drag element, is conceivable, but promises many experimental difficulties. "22

Also,

"Temperature gradients clearly depend on a great many of test conditions, including detailed model configurations. ${ }^{3}$

- French researchers have also studied the thermal gradient problem. They dismiss using temperature sensors to solve the problem:

"Furthermore, the correction of these effects, calculated from measurements of point temperatures performed by thermocouples, appeared as very unreliable and unrepeatable for large integral balances as the thermocouples, although numerous, insufficiently analyze the thermal variations occurring within the structure." ${ }^{4}$ Instead of using temperature sensors to compensate for axial strain output due to thermal gradients, DuBois and others propose measuring the force due to temperature gradients using a second strain-gage bridge. They state, qualitatively, that their results with the second bridge show better thermal gradient compensation with less time lag and less hysteresis.

\subsection{Current NTF Balance Configuration}

Current NASA-LaRC NTF cryogenic state of the art internal balances are fabricated from VascoMax C-200 (Teledyne Vasco, 18\% Nickel maraging steel). These 
monolithic balances require intricate machining in order to gain the required resolution and accuracy of all forces and moments in six degrees of freedom as well as carry all aerodynamic loads induced on the model to the tunnel support structure.

These balances are instrumented with twelve $350 \Omega$ foil resistive strain gage bridges, a complete primary and redundant set of component measurements, and nine 100 $\Omega$ platinum resistive temperature detectors. The individual strain gages are cryogenically tested and matched into optimum bridge configurations prior to installation on the actual balance. After strain gage installation the isothermal temperature effects on the electrical zero of the balance is compensated by installing a section of temperature sensitive wire within the proper leg of the strain gage bridge. This compensation only addresses steady state isothermal temperature changes of the balance, not steady state or transient thermal gradient effects. The design of the strain gage layout is optimized to minimize thermal gradient effects.

\subsection{Impact of thermal gradients on wind tunnel data acquisition}

Uncorrected thermal gradients on the balance do affect the quality of axial force (drag) measurements. For example, current NTF balances have uncorrected thermal effects of approximately $1 \%$ of the full-scale load. (A cryogenic balance for the NTF has a typical accuracy of $.25 \%$ of the full-scale load so any thermal effects are desired to be within this range to determine accurately the aerodynamic loads on the model.) The goal is for the balance accuracy to be $0.125 \%$ of full-scale load so uncompensated outputs can be as much as eight times the specified accuracy of the balance.

In addition, the potential for thermal gradients affects wind tunnel productivity. In order to reduce the magnitude of the gradients, the users of the tunnel usually wait till the balance is in within a specified temperature range before taking data. For NTF, the temperature range is set at $5.6^{\circ} \mathrm{C}\left(10^{\circ} \mathrm{F}\right)$, a fairly conservative value. Thus, the tunnel is "running" without any data being recorded. The presence of thermal gradients also necessitates the extra "wind-off zero" measurements that slow wind tunnel productivity.

Compensating for thermal gradients requires either (a) temperature sensors on the balance or (b) extra strain gages on the balance or both. The extra sensors increase the number of wires on the balance thus increasing the potential for a parallel load path. The extra sensors also increases the complexity of the data acquisition system.

\subsection{Present thermal gradient compensation techniques}

Presently there are procedures used at NASA-LaRC to account for the strain due to thermal gradients. Hence, NTF does produce reliable data. The biggest drawback to present techniques is that they do not fully correct for all gradient conditions (profiles).

The most complex thermal gradient compensation techniques are required for the axial force component. The balance has a primary (AFB1) and a secondary (AFB2) axial 
force strain gage bridge. These bridges (AFB1 and AFB2) are completely electrically isolated from each other and are gaged in symmetric locations on the balance to provide identical resolution and accuracy. AFB1 utilizes a thermal gradient compensation technique developed by NASA-LaRC in which temperature sensitive Nickel wire is installed in four strategic locations on the balance structure and is wired into two opposing legs of the strain gage bridge. This technique requires an iterative process of repeated temperature excursions and adjustment of the sensor's position and resistance. AFB2 utilizes the same strategy for thermal compensation except instead of Nickel sensors, which are integrated electrically into the strain gage bridge, four of the nine platinum temperature sensors are strategically placed on the balance structure and the compensation technique derives an algorithm which is performed analytically within the software of the data acquisition system. This approach provides significant improvement to the reliability and ruggedness of the balance as well as reduces the number of cryogenic excursions required to perform the thermal gradient compensation.

Both of the thermal compensation techniques which are currently in use do not fully compensate the effects on the axial force component for any gradient profile or magnitude. Wind tunnel testing procedures such as frequent wind off zeroes and active temperature conditioning of the balance are utilized in order to minimize the effects of thermal gradients. The residual thermal gradient effects, which are currently uncompensated for, have a direct effect on tunnel productivity and data quality.

\section{Thermally-Induced Gradient Effects Research (TIGER)}

\subsection{Overview of TIGER}

To address the thermal gradient issue, NASA-LaRC has initiated a research program called Thermally-Induced Gradient Effects Research (TIGER). The approach in TIGER is to generate various steady state and gradient temperature profiles on a speciallyprepared "research" balance. The research balance is instrumented with 118 temperature sensors, 64 strain gages, and 40 foil resistive heater elements. This balance will then be subjected to a variety of temperature and load configurations. The ultimate goals of the TIGER program are to (a) understand the physics of the thermally-induced strain and its subsequent impact on load measurements and (b) develop a robust thermal gradient compensation technique.

A key challenge in the TIGER program is how to analyze the data from the special research balance. Considering just all combinations of temperature sensors leads to more than $3.6 \times 10^{35}$ possible combinations and that does not even include adjustments to the sensor weights. Clearly, an exhaustive search to find the best combination is unfeasible.

Four data analysis techniques were considered: linear regression, neural network, eigenvector decomposition, and genetic algorithms. The final recommendation, after considering advantages and disadvantages of each technique, was to use a genetic 
algorithm (GA). The GA was chosen because of its suitability to the task of finding the optimal or near-optimal solution in a multivariable search space.

\subsection{Description of GA}

Genetic algorithms are used to find a global maximum or minimum in a search space. ${ }^{5}$ Unlike other search mechanisms that sample the search space at one point and then move to a better point based on the slope (gradient) information at that point, genetic algorithms work with a population which is a sampling of several points. Each point that is sampled is assigned a "fitness" value. Fitness is simply a number that portrays how useful that point is in solving the problem at hand. The "points" are actually strings or sets of characteristics. For example, a string could be the weights applied to the temperature sensors.

Once each string is assigned a fitness value, the next population is produced by the operations of selection, crossover, and mutation. Selection means that those strings with the greatest fitness values survive. Crossover is the recombination of traits of the selected strings; it is accomplished by swapping parts of randomly-selected strings. Mutation randomizes small parts of the resulting strings.

\subsection{Application of GA to TIGER}

The overall strategy is to define a "string" or weight vector that specifies the weight to apply to each temperature sensor on the balance. For example, if the weight is 0 then that sensor is not used. If the weight is +1 then the result from that sensor is added. If -1 , then the result is subtracted. Finally, we solve to determine the weight vector that gives the best performance.

We define best performance as the combination of weights that has the greatest impact on the apparent strain. This impact will be quantified using the correlation between the (weighted) temperature measurement(s) and the output from the bridge.

An important aspect of GA design is the choice of the fitness function. A fitness function is how the algorithm determines whether one potential solution is better than another. We use a fitness function of the form

$$
\mathrm{F}=e^{-(A+B)^{2}} \text {. }
$$

With this fitness function, we want $\mathrm{A}$ and $\mathrm{B}$ to be small (near 0 ) so that $\mathrm{F}$ is large (near 1 ). $\mathrm{A}$ and $\mathrm{B}$ are parameters that we want to minimize.

\section{(a) Calculation of A coefficient}

We use the A coefficient to steer the GA towards solutions that have a minimum number of terms. In this way, we can limit the instrumentation cost of outfitting each balance with a multitude of sensors. The A coefficient is given by ${ }^{6}$ 


$$
A=\frac{\text { number_actual }- \text { number_desired }}{A_{-} \text {width }},
$$

where

$$
\begin{aligned}
& \text { number_actual }=\text { number of non-zero temperature weights } \\
& \text { number_desired }=\text { desired number of non-zero weights (user-specified) }
\end{aligned}
$$$$
\text { A_width = user-defined input to specify how much to steer towards fewer }
$$

terms

Since test data is presently only available for a standard balance with nine temperature sensors, the A coefficient was not investigated in the preliminary results.

(b) Calculation of B coefficient

The B coefficient will be based on the correlation coefficient, $\rho$, between the weighted temperature measurement and the apparent strain (Wheatstone bridge output).

$$
B=B_{-} \text {width }(1-\rho)
$$

The computationally-intensive part is the calculation of $\rho$. Specifically,

$$
\begin{aligned}
& \rho=E\left\{\left(\frac{A F B-\overline{A F B}}{\sigma_{A F B}}\right)\left(\frac{W T-\overline{W T}}{\sigma_{W T}}\right)\right\} \\
& =\frac{1 /(N-1) \sum_{i=1}^{N}\left(A F B_{i}-\overline{A F B}\right)\left(W T_{i}-\overline{W T}\right)}{\sigma_{A F B} \sigma_{W T}}
\end{aligned}
$$

In the above equations, WT and AFB are random variables that correspond to weighted sum of the temperature measurements and the axial force bridge output, respectively. $E\{\}$ represents the expectation operator.

The correlation coefficient, $\rho$, varies from -1 to +1 . If $\rho$ is near 1 , then the weighted temperature measurements, WT, vary in sync with axial force measurement, AFB. If $\rho$ is near zero, then there is no pattern to how the two signals vary in relation to each other.

To implement the GA, we compiled and linked over 50,000 lines of $\mathrm{C}++$ code. A majority of the code was available (free of charge) through MIT. We installed the code, debugged it, developed a makefile to link together all the libraries and added several routines to use it for TIGER.

\subsection{Results from GA}


The GA was tested on data from a standard balance with 9 temperature sensors. The balance was subjected to a temperature cycle from room temperature up to about $65^{\circ}$ $\mathrm{C}\left(150^{\circ} \mathrm{F}\right)$ and then down to $-185^{\circ} \mathrm{C}\left(-300^{\circ} \mathrm{F}\right)$. During this time, the axial force bridge (AFB) was monitored. This temperature cycle was repeated in the Model Prep Area of the NTF.

The output from a temperature cycle is shown in Figure 2. Note that there is no external force acting on the balance so that any change in AFB output must be due to environmental conditions. The laboratory data shows variations in AFB of about \pm 20 $\mu \mathrm{V} / \mathrm{V}$, which corresponds to $4 \%$ of full-scale load. Hence, thermal cycling alone causes variations that are over 10 times the specified accuracy of the balance. Since corrections are made for any uniform rise in temperature, we assume that all variations in AFB are due to thermal gradients.

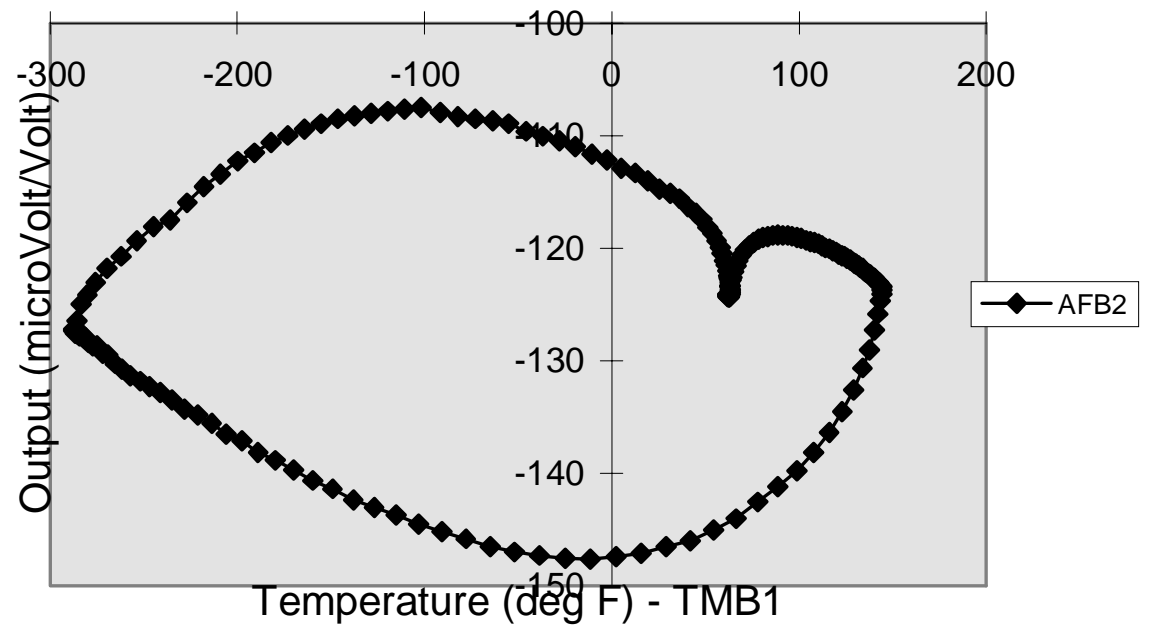

Figure 2: Sample output of axial force bridge output during cryogenic temperature excursion.

The GA was used to find a set of weights, $\mathrm{w}_{\mathrm{i}}$, to apply to the temperature sensors to correct for the variations in the AFB. We applied the weights to the temperature measurements to form WT, the weighted temperature measurement:

$$
\mathrm{WT}=\mathrm{W}_{1} \mathrm{~T}_{1}+\mathrm{W}_{2} \mathrm{~T}_{2}+\ldots+\mathrm{W}_{9} \mathrm{~T}_{9} .
$$

As noted in Section 3.3, the criteria for the best set of weights was that set of weights that resulted in WT having the highest correlation with the AFB.

Once the GA calculates the weights that give the highest correlation with the AFB, a corrected output can be found using 


$$
\mathrm{AFB}(\text { compensated })=\mathrm{AFB}(\mathrm{raw})-\frac{\sigma_{A F B}}{\sigma_{W T}}\left(\mathrm{WT}-\mathrm{WT}_{\mathrm{AVG}}\right) .
$$

In the above equation sigma represents the standard deviation of the AFB and WT measurements, respectively. A plot of AFB(compensated) is shown in Figure 5. For comparison the raw AFB (AFB2, diamond shapes) is also plotted. Clearly, the variation in AFB due to thermal gradients has been reduced. The compensated AFB output, $\mathrm{AFB}$ (compensated), varies by only $\pm 1.5 \mu \mathrm{V} / \mathrm{V}$, which is a 10 times reduction in variation. Also shown on the plot (dotted lines) is the output that would occur if the on-board correction only was used.

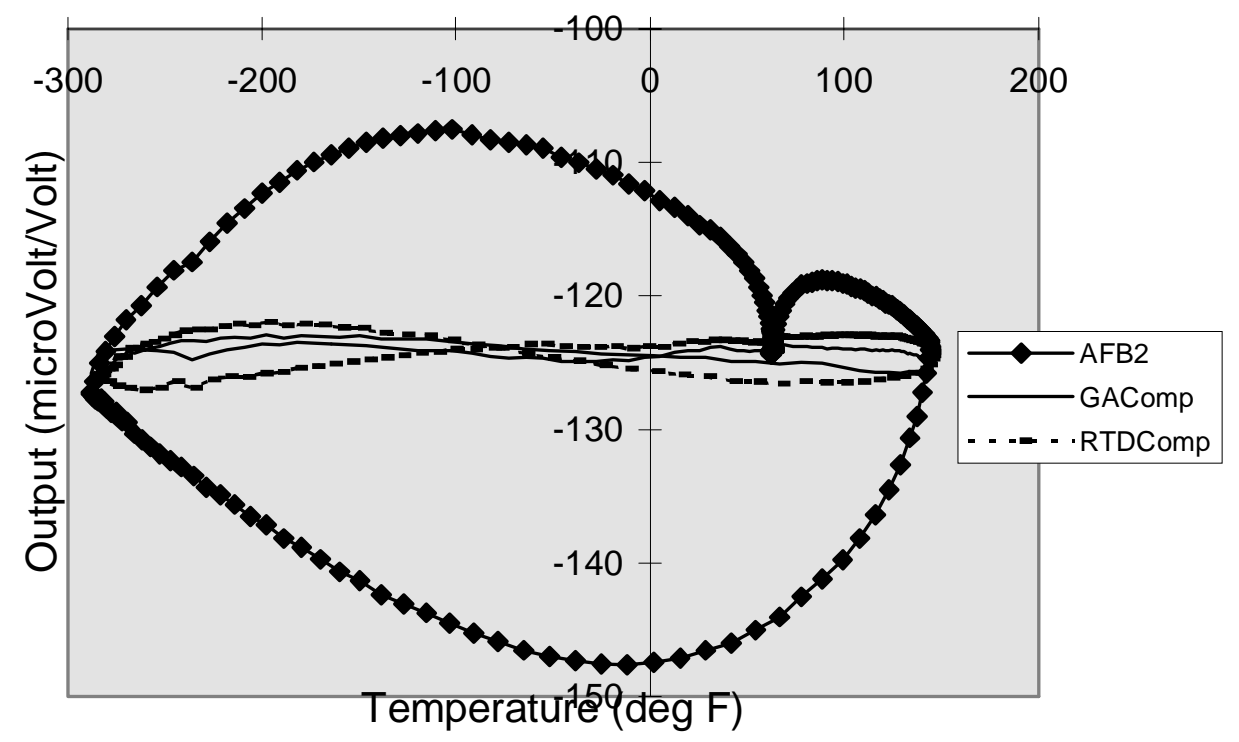

Figure 3: AFB(raw) shown by AFB2 curve with diamond shapes, AFB compensated with GA-derived weights (GAComp), and AFB compensated with onboard correction (RTDComp).

\subsection{Issues with the GA results}

Two main issues came out of the GA results. The first issue we encountered was why the same input data would give different output results (i.e., different set of weights). In this case, the output was not unique. Common sense implies that the same input data should result in the same output data. The second issue arose when we tried to "test" the weights. That is, we developed a set of weights for the temperature sensors using the GA on lab data. We then applied those weights to data from the Model Prep Area but there was no improvement in the AFB response. In fact, the AFB curve showed greater variation when subjected to thermal gradients. Thus, the weights were not robust or transferable to different test conditions.

\subsubsection{Uniqueness}


The non-uniqueness issue is a problem with the search space and not with the implementation of the GA. In the experiment, the nine temperature measurements are highly correlated with each other. This leads to a very flat search space, since correlation with the axial force output is the figure of merit. This is illustrated pictorially in figure 4 . Figure (a) shows a well-defined maximum peak. Any optimization method can easily find the single peak. Figure (b), on the other hand, has a flat maximum - several points in the search space give the same output. In the thermal gradient compensation algorithm, several combinations of sensors have equal correlation with the axial force output. Since the GA used a random starting seed, it found a different solution each time, though each solution was equally valid.
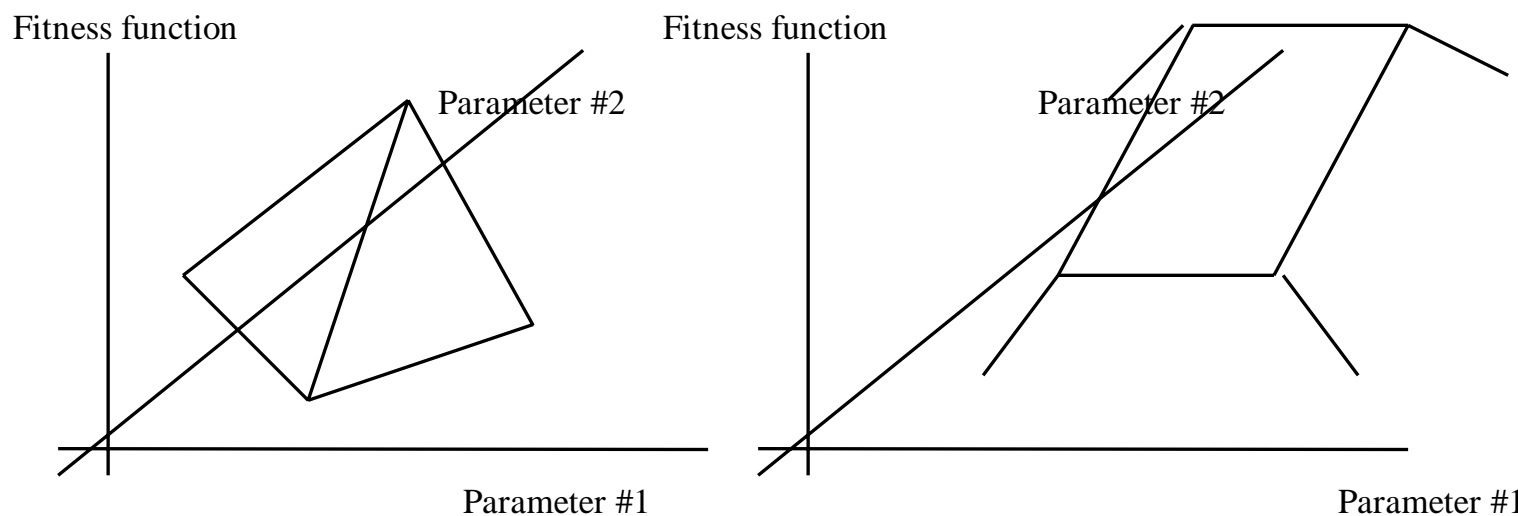

Figure 4.: Examples assuming a 2 parameter search space. (a) A single, clear maximum value for the fitness function is apparent; (b) Several values of the two parameters lead to equally high values for the fitness function.

To overcome the uniqueness issue, more constraints must be added to the fitness function of the GA.

\subsubsection{Transference of weights}

Several hypotheses have been suggested for why the results from the lab data set did not apply to the case when a model is on the balance. Note that this was not just an issue with the GA; results from an exhaustive least squares search also had the robustness/transference problem. The hypotheses included (a) the GA did not find the true maximum, (b) temperature and strain measurements were not made at the same time, (c) there are not enough temperature sensors and/or they are not in the correct locations, (d) there are anomalies in the data (e.g., thermal emfs, parallel loadpaths) that affect the axial force output, and (e) an incorrect mathematical model is being used. Hypotheses (a) and (b) were checked and discounted.

One assumption (hypothesis (e)) with the GA was that a linear combination of temperature measurements would give an accurate representation of the thermal gradients 
that affect the axial force output. To check that assumption, we tried several different mathematical models. We developed a figure of merit that measured how well each model compensated for thermal gradient effects. We then trained each model on data from the laboratory and applied that model to data from the Model Preparation Area (MPA) at NTF.

Results were compiled for the following mathematical models: linear, sin, exponential, natural log, second order, linear plus second order, and various autoregressive moving-average (ARMA) models. The ARMA model gave excellent results but it utilizes past output and input data samples; it was thus impractical to implement in a wind tunnel environment since it would also cancel any constant applied load on the balance. Of the other models, only the linear model gave decent results when applied to the data set from the MPA. Thus, we conclude that it is the best model.

The result of the mathematical model investigation narrowed the number of hypotheses to two: either there are some data anomalies or the thermal gradients are too complicated to be completely mapped with nine temperature sensors. Testing with the research balance will allow us to distinguish between the two hypotheses. In either case, the lack of transferability/robustness is not an artifact of the GA.

\section{Summary}

1. Thermal gradients on the force measurement balance are a problem with getting accurate data at high Reynolds' numbers in a cryogenic wind tunnel.

2. Thermal gradients reduce wind tunnel productivity.

3. Finding a thermal compensation algorithm is difficult due to (a) complex thermal flowpath through the balance, (b) complex force flowpath through the balance, (c ) corresponding uncertainty of where to place the temperature sensors, (d) high correlation of temp measurements causes several numerical solution techniques to break down due to singular matrices (divide-by-0 errors), (e) unknown differences in data acquisition set-up between the laboratory, the ready bay and the wind tunnel.

4. The special-purpose research balance is a tool for determining the severity and impact of thermal gradients on wind tunnel measurements. NASA-Langley is in the process of developing software to analyze data from the research balance.

5. The impact of thermal gradients is very complex. Continued investigation is required.

\footnotetext{
${ }^{1}$ G.F. Moss and D. G. Payne, "A Compact Design of Six Component Internal Strain Gauge Balance", Roayl Aircraft Establishment, Technical Note No. AERO. 2764, July 1961, Appendix 1.
} 
2 J. F. Baljeu, "Development of a Multi-Component Internal Strain-Gauge Balance for Model Tests in a Cryogenic Wind Tunnel", National Aerospace Laboratory, Report \# NLR TR 88157 U, October 30, 1988, pg. 17.

${ }^{3}$ Ibid, pg. 27.

${ }^{4}$ M. DuBois, " Six-Component Strain-Gage Balances for Large Wind Tunnels," $4^{\text {th }}$ International Congress on Experimental Mechanics, May 1980, pg. 7.

${ }^{5}$ John H. Holland, "Genetic Algorithms", Scientific American, July 1992, pp. 66 - 72.

${ }^{6}$ Sigurd A. Nelson, "Strain Gage Selection in Loads Equations Using a Genetic Algorithm", NASA Contractor Report 4597, 1994. 